\title{
New Compound Upper Bound on MIMO Channel Capacity
}

\author{
Sergey Loyka, Member, IEEE, and Ammar Kouki, Senior Member, IEEE
}

\begin{abstract}
MIMO channel capacity may be severely degraded due to correlation between individual sub-channels of the matrix channel. Several models, which are limited to some specific scenarios, have been developed to date to account for this effect. In this letter, we derive a new upper bound on the mean (ergodic) MIMO capacity, which is not limited to a particular scenario and accounts for both transmit $(\mathbf{T x})$ and receive ( $R x)$ end correlations in such a way that their impact can be estimated separately and compared. Thus, a conclusion can be made as to which end contributes more to capacity reduction. In general, the higher correlated end has a dominant effect on the capacity.
\end{abstract}

Index Terms-Channel capacity, correlation, MIMO.

\section{INTRODUCTION}

$\mathbf{M}$ ULTIPLE-INPUT multiple-output (MIMO) communication architecture has recently emerged as a new paradigm of spectrum-efficient wireless communications in rich multipath environment [1]. Unprecedented wireless spectral efficiencies, ranging from $20-40 \mathrm{bit} / \mathrm{s} / \mathrm{Hz}$, have been demonstrated in a laboratory environment [2] with this architecture. Even higher spectral efficiencies may be achieved in certain environments when the system design is optimal. However, under real-world conditions the MIMO channel capacity may be limited due to several factors. One of the most important such factors is the correlation between sub-channels of the matrix channel [3], [4]. For a completely uncorrelated matrix channel, the MIMO capacity reaches its maximum and scales roughly linearly as the number of antennas. The correlation between individual receive and/or transmit branches results in capacity reduction. Several models have been used to study and quantify this phenomenon but have always been limited to some specific scenarios [3], [9], [11]. In particular, the one-ring scattering model has been used in [3] to derive the lower and upper bounds on the mean MIMO capacity. While providing useful insight, these bounds are limited to the case of one-end (i.e., either Tx or Rx) correlation only. A more general MIMO capacity analysis in a correlated channel, which is basically a generalization of the earlier work by Telatar [8], [13], has been reported in [11]. The analysis in [11] accounts for both Tx and $\mathrm{Rx}$ end correlations using the eigenvalue decomposition technique. However, crucial limiting assumptions of the analysis

Manuscript received November 13, 2001. The associate editor coordinating the review of this letter and approving it for publication was Prof. K. Kiasaleh.

S. Loyka is with the School of Information Technology and Engineering (SITE), University of Ottawa, Ottawa, ON K1N 6N5, Canada (e-mail: sergey.loyka@ieee.org).

A. Kouki is with the Department of Electrical Engineering, Ecole de Technologie Superieure, 1Montreal, QC H3C 1K3, Canada..

Publisher Item Identifier S 1089-7798(02)02981-2. above are that the channel correlation matrix can be factorized into a product of Tx and Rx end correlation matrices (resulting in separate eigenvalues for the Tx and Rx ends) and that the number of antennas is large (ideally infinite). This is not the case in many practically-important scenarios (e.g., indoor or measured channels or any system with a moderate number of antennas). Besides, the eigenvalue decomposition techniques is used in [11] that limits the physical insight provided because no simple analytical models are available for the eigenvalues and the resulting estimation is rather complex mathematically and can be implemented only through numerical analysis.

In this letter, we derive a universal upper bound on the mean (ergodic) MIMO channel capacity, which is independent of the scenario considered and accounts for both the Tx and Rx end correlations, using Jensen's inequality and concavity of the $\log$ det function. No assumption of the channel correlation matrix factorization is made. The bound is expressed in terms of the Tx and Rx correlation matrices in such a way that the comparison of the Tx and Rx end contributions to the capacity reduction can be made in a general case. The well-known correlation matrix models developed for the diversity combining systems can also be applied to the MIMO system analysis using our approach [12].

The main goal of this paper is to study general properties of the MIMO capacity rather than to estimate it accurately in some specific scenarios. In particular, we show- to the best of our knowledge - for the first time, that the direct application of Jensen inequality does not capture the transmit end correlation, and we propose a method to accomplish this.

\section{MIMO CHANNEL CAPACITY}

For a fixed linear $m \times n$ matrix channel with additive white Gaussian noise and when the transmitted signal vector is composed of statistically independent equal power components each with a Gaussian distribution and the receiver knows the channel, the channel capacity is [1]

$$
C=\log _{2} \operatorname{det}\left(\mathbf{I}+\frac{\rho}{n} \mathbf{H} \cdot \mathbf{H}^{+}\right) \text {bits } / \mathrm{s} / \mathrm{Hz}
$$

where $n$ and $m$ are the numbers of transmit and receive antennas respectively, $\rho$ is the average signal-to-noise ratio, $\mathbf{I}$ is $m \times m$ identity matrix, $\mathbf{H}$ is the normalized channel matrix, which is considered to be frequency independent over the signal bandwidth, and "+" denotes transpose conjugate. When the channel is random (stochastic), then the capacity is random, too. The mean (ergodic) capacity can be defined in this case as [8], [13]

$$
\langle C\rangle=\left\langle\log _{2} \operatorname{det}\left[\delta_{i j}+\frac{\rho}{n} \cdot r_{i j}\right]\right\rangle
$$


where $r_{i j}$ is "instantaneous" correlation matrix (i.e., for a given channel realization)

$$
r_{i j}=\sum_{k} h_{i k} h_{j k}^{*}
$$

$\delta_{i j}$ is Kroneker's delta, \langle\rangle is the expectation over the channel matrix, and $h_{i j}$ denotes the components of $\mathbf{H}\left(h_{i j}\right.$ is the transfer factor between $j$ th transmit antenna and $i$ th receive antenna). Note that Eq. (2) does take into account correlation occurring at both the transmit and receive ends. This equation can be used for statistical simulations to evaluate $\langle C\rangle$ for some specific models of the channel matrix. However, these matrix numerical computations can be very lengthy, especially when the number of antennas is very large. Thus, a computationally efficient technique is desirable. Another difficulty with (2) is that it includes the impact of receive and transmit end correlation in an integrated form. Hence, it is not easy to decide in general which end contributes more to capacity reduction.

To this end, Jensen's inequality can be used to obtain an upper bound on $\langle C\rangle$. According to this inequality and concavity of $\log$ det function [5], one obtains

$$
\langle C\rangle \leq \overline{C_{R}}=\log _{2} \operatorname{det}\left[\delta_{i j}+\frac{\rho}{n} \cdot r_{i j}^{R}\right]
$$

where $r_{i j}^{R}$ is the correlation matrix of the Rx branches

$$
r_{i j}^{R}=\sum_{k}\left\langle h_{i k} h_{j k}{ }^{*}\right\rangle .
$$

Thus, using (4) and some models of the correlation matrix (see, for example, [6], [7]), one may evaluate the upper capacity bound. However, it should be noted that the correlation matrix in (5) does not capture the correlation of the transmit branches since $k$ represents the Tx antenna index and it is the same for both factors. Hence, the upper bound in (4) can be close to the mean capacity when the correlation of the Rx branches is much higher than that of the Tx branches. However, if the Tx branch correlation is higher than the Rx one, the upper bound in (4) is not an accurate approximation of the mean capacity. Therefore, in order to have an upper bound that is as close as possible to the mean capacity, one must also account for the transmit correlation. To this end, the reciprocity of (1) can be used in the following way. First, we note that the MIMO capacity given by (1) is invariant under the transformation $\mathbf{H} \rightarrow \mathbf{H}^{T}$, where " $T$ " means transpose [this follows from the matrix identity $\operatorname{det}(\mathbf{I}+\mathbf{A} \cdot \mathbf{B})=\operatorname{det}(\mathbf{I}+\mathbf{B} \cdot \mathbf{A})]$. This in effect is equivalent to reversing the direction of information transmission. Thus, (2) still holds true if we define $r_{i j}$ as

$$
r_{i j}=\sum_{k} h_{k i} h_{k j}{ }^{*} \text {. }
$$

Hence, one obtains the second upper bound (the transmit bound)

$$
\langle C\rangle \leq \overline{C_{T}}=\log _{2} \operatorname{det}\left[\delta_{i j}+\frac{\rho}{n} \cdot r_{i j}^{T}\right]
$$

where $r_{i j}^{T}$ is the correlation matrix of the Tx branches

$$
r_{i j}^{T}=\sum_{k}\left\langle h_{k i} h_{k j}{ }^{*}\right\rangle .
$$

It is interesting to note that while $\mathbf{H} \rightarrow \mathbf{H}^{T}$ does not change the mean capacity, it does change the upper bound in the most desirable way. It should also be emphasized that the upper bound in (7) does not capture the Rx branch correlation. Therefore, this upper bound will be close to the mean capacity when the transmit correlation is higher than the receive one. However, if the opposite is true, then this upper bound is not an accurate approximation of the mean capacity.

From inequalities (4) and (7) it is clear that a tighter upper bound of the mean channel capacity can be obtained by combining them. Thus, we form the compound upper bound by taking minimum of the two bounds defined above (because the effect of correlation is to decrease capacity)

$$
\overline{C_{c}}=\min \left[\overline{C_{R}}, \overline{C_{T}}\right] .
$$

This upper bound is much tighter than the receive or transmit bound considered separately when the transmit and receive branch correlations are significantly different.

It should be mentioned that the Jensen inequality has been earlier applied to the MIMO capacity analysis in [3]. However, the method in [3] is limited to the case of one-end (i.e., Tx or Rx but not both) correlation only. Our method accounts for both Tx and Rx end correlations without any limiting assumptions (e.g., the channel matrix factorization in [11]).

Let us now consider an illustrative example of a correlated Rayleigh channel with correlation occurring at both the Tx and Rx ends. The components of $\mathbf{H}$ are taken to be identically distributed correlated complex Gaussian variables (real and imaginary parts are identically distributed and independent, i.e., the phase is uniformly distributed over $[0,2 \pi]$ ) with zero mean and unit variance. The correlation matrix of $\mathbf{H}$ is assumed to be of the following form:

$$
R_{i j, k l}=\left\langle h_{i k} h_{j l}{ }^{*}\right\rangle=R_{i j}^{R} \cdot R_{k l}^{T}
$$

where $R_{i j}^{R}$ and $R_{k l}^{T}$ are the correlation matrices of the receive and transmit branches correspondingly. The factorization in (10) can be justified when the Tx correlation is introduced by local scatterers around the Tx end and is independent of the Rx correlation, which, in turn, is introduced by local scatterers around the Rx end [9], [10]. We adopt further the uniform correlation matrix model for $R_{i j}^{R}$ and $R_{k l}^{T}$, which provides the worst-case estimation [4]

$$
R_{i j}^{R}=\left\{\begin{array}{ll}
r, & i \neq j \\
1 & i=j,
\end{array} \quad R_{k l}^{T}= \begin{cases}1-r, & k \neq l \\
1 & k=l\end{cases}\right.
$$

where $0 \leq r \leq 1$. Fig. 1 shows the mean capacity of this channel, obtained by extensive Monte Carlo simulations (2), and the receive (4), transmit (7) and compound (9) bounds. In this example, $r=0$ corresponds to fully uncorrelated $\mathrm{Rx}$ branches and full correlation of the Tx ones; $r=1$ corresponds to full correlation of the Rx branches and fully uncorrelated Tx ones. The compound bound provides a good approximation to the mean capacity when the effect of correlation is significant (i.e., $r \leq 0.2 \cdots 0.3$ and $r \geq 0.7 \cdots 0.8$ ) while the receive or transmit bounds alone are not accurate. The compound upper bound is 


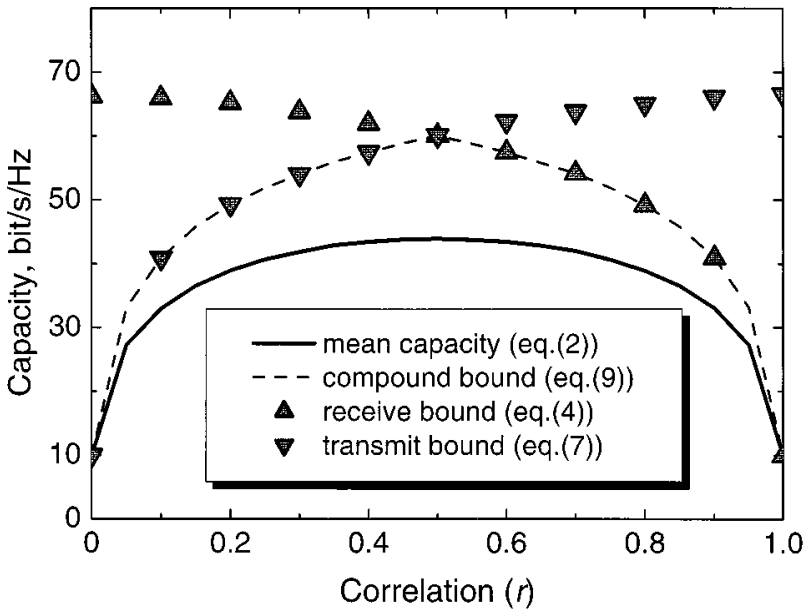

Fig. 1. MIMO capacity of a correlated Rayleigh channel for $m=n=10$ and $\rho=30 \mathrm{~dB}$.

rather loose when $r$ is around 0.5. However, the impact of correlation in this case is small and the MIMO capacity can be estimated in a well-known way [1], [8], [13]. The computational efficiency of upper bound evaluation is a few orders of magnitude higher than that of the mean capacity. It is also interesting to note that the maximum capacity is achieved for $r=0.5$. This indicates that decrease in capacity is usually due to that end (transmit or receive) which has higher correlation. Thus, a rough estimation of the capacity may be obtained by considering only the higher correlated end.

\section{CONCLUSION}

In this letter, we presented a new compound upper bound on the mean (ergodic) MIMO channel capacity, which accounts for both transmit and receive end correlation in such a way that their impact can be estimated separately and without any additional limiting assumptions. Thus, a conclusion can be made as to which end contributes more to capacity reduction, which is not easy to do using traditional methods. The compound upper bound is tighter than the transmit or receive bounds alone and is not limited to some particular scenarios. It can be used for an approximate estimation of the MIMO capacity using the correlation matrix models developed for space-diversity techniques [6], [7], [12]. The upper bound is shown to follow the same dependence on correlation as the mean capacity does. Hence, two different scenarios (or the impact of Tx and Rx ends) can be compared using the difference upper-bounded capacity. It is also shown that the higher correlated end has a dominant effect on the MIMO capacity.

\section{REFERENCES}

[1] G. J. Foschini and M. J. Gans, "On limits of wireless communications in a fading environment when using multiple antennas," Wireless Pers. Commun., vol. 6, no. 3, pp. 311-335, Mar. 1998.

[2] G. D. Golden, G. J. Foschini, R. A. Valenzuela, and P. W. Wolniansky, "Detection algorithm and initial laboratory results using V-BLAST space-time communication architecture," Electron. Lett., vol. 35, no. 1, pp. 14-16, Jan. 1999.

[3] D. S. Shiu, G. J. Foschini, M. J. Gans, and J. M. Kahn, "Fading correlation and its effect on the capacity of multielement antenna systems," IEEE Trans. Commun., vol. 48, pp. 502-513, Mar. 2000.

[4] S. L. Loyka and J. R. Mosig, "Channel capacity of N-antenna BLAST architecture," Electron. Lett., vol. 36, no. 7, pp. 660-661, Mar. 2000.

[5] T. M. Cover and J. A. Thomas, Elements of Information Theory. New York: Wiley, 1991.

[6] J. Salz and J. H. Winters, "Effect of fading correlation on adaptive arrays in digital mobile radio," IEEE Trans. Veh. Technol., vol. 43, pp. 1049-1057, Nov. 1994.

[7] W. C. Y. Lee, Mobile Communications Engineering. New York: McGraw Hill, 1998.

[8] I. E. Telatar, "Capacity of multi-antenna Gaussian channels,", AT\&T Bell Lab. Internal Tech. Memo., June 1995.

[9] D. Chizhik et al., "Effect of antenna separation on the capacity of BLAST in correlated channels," IEEE Commun. Lett., vol. 4, pp. 337-339, Nov. 2000

[10] A. L. Moustakas et al., "Communication through a diffusive medium: Coherence and capacity," Science, vol. 287, pp. 287-290, Jan. 2000.

[11] A. M. Sengupta and P. P. Mitra, "Capacity of multivariate channels with multiplicative noise: I. Random matrix techniques and large- $N$ expansions for full transfer matrices," Bell Labs., Tech. Rep., 2001.

[12] S. Loyka and A. Kouki, "The impact of correlation on multi-antenna system performance: Correlation matrix approach," in 2001 IEEE Vehicular Technology Conf., Atlantic City, NJ, Oct. 7-11, pp. 533-537.

[13] I. E. Telatar, "Capacity of multi-antenna Gaussian channels," Eur. Trans. Telecom., vol. 10, no. 6, Dec. 1999. 